\title{
Microarray analysis of the aberrant microRNA expression pattern in gliomas of different grades
}

\author{
XIAO-PENG ZHU ${ }^{1 *}$, KE-JIE MOU ${ }^{1 *}$, QING-FU XU ${ }^{1}$, JUN-HAI TANG $^{1}$, GUO-HAO HUANG ${ }^{1}$, JIAN-PING XU ${ }^{2}$, \\ GUANG-HUI LI ${ }^{3}$, SI-JIN AI ${ }^{4}$, JEAN-PHILLIPPE HUGNOT ${ }^{5}$, ZHENG ZHOU ${ }^{1}$ and SHENG-QING LV ${ }^{1}$
}

Departments of ${ }^{1}$ Neurosurgery and ${ }^{2}$ Pathology, and ${ }^{3}$ Institute of Cancer, Xinqiao Hospital, Third Military Medical University, Chongqing 400037; ${ }^{4}$ Department of Neurosurgery, China People's Liberation Army No. 94 Hospital, Nanchang, Jiangxi 330026, P.R. China; ${ }^{5}$ INSERM U1051, Institut des Neurosciences de Montpellier, 34091 Montpellier, France

Received January 23, 2015; Accepted April 17, 2015

DOI: $10.3892 /$ or.2015.3953

\begin{abstract}
Previous studies have focused on miRNA expression in brain gliomas. However, both the expression pattern of miRNAs in gliomas of different grades and various miRNAs involved in malignant progression of gliomas are poorly understood. In the present study, we used miRNA microarraybased screening to investigate the miRNA expression profile in gliomas, which was further verified by qRT-PCR in selected miRNAs. In total, we found 13 differentially expressed miRNAs between gliomas and their matched surrounding tissues. Among them, 12 miRNAs were upregulated and only one (miR-4489) was downregulated compared with the control. Furthermore, the lower expression level of miR-4489 was confirmed by qRT-PCR in 26 glioma samples. Our microarray result revealed 8,9 and 15 aberrantly expressed miRNAs in gliomas of World Health Organization (WHO) grade II-IV, respectively. Gene Ontology (GO) and Pathway analysis indicated that target genes of the 13 miRNAs were significantly enriched in central nervous system- and tumor-related biological processes and signaling pathways. The dysregulated miRNAs identified in the present study contribute to the tumorigenesis and malignant progression of gliomas and may serve as useful markers for advanced glioma pathological grading and prognosis.
\end{abstract}

\section{Introduction}

Brain gliomas are the most common primary intracranial tumors. Malignant gliomas are often characterized by inva-

Correspondence to: Dr Zheng Zhou or Dr Sheng-Qing Lv, Department of Neurosurgery, Xinqiao Hospital, Third Military Medical University, Chongqing 400037, P.R. China

E-mail: m13808337911_1@163.com

E-mail: lvsq0518@hotmail.com

${ }^{*}$ Contributed equally

Key words: microRNAs, microarray, gliomas, grade, prognosis sive growth, a high rate of relapse and rich vascularity (1). Despite advances in treatment modalities and the meaningful benefits in regard to survival (the median survival in glioblastoma increased from 12.1 to 14.6 months with surgical resection followed by radiotherapy and temozolomide chemotherapy) (2), the overall outcome of gliomas remains extremely unsatisfactory. According to the World Health Organization (WHO) classification of central nervous system tumors, brain gliomas are classified into grades I-IV, which partially indicate the degree of malignancy and prognosis (3). However, even gliomas with the same grade or histological type do not have identical prognosis. For example, primary and secondary glioblastoma are both included in WHO IV and they cannot be distinguished by histopathology; however, the incidence, onset age, survival rate and gene expression profiles are quite different (4). Thus, it is critical to illuminate the mechanisms underlying the development and progression of gliomas and detect sensitive and specific biomarkers for diagnosis, grading and prognosis of the disease.

MicroRNAs (miRNAs) are emerging as an important class of endogenous small ( $22 \mathrm{nts})$ non-coding RNAs. They have the ability to negatively regulate gene expression at the post-transcriptional level by binding to the complementary sequence in the 3'-untranslated region (UTR) of target mRNAs (5). Aberrant expression of miRNAs has been found in a wide variety of human tumors, including brain gliomas. Most miRNAs have been shown to act as either oncogenes or tumor suppressors (6). Since the first identification of dysregulated miRNA-21 in human gliomas (7), a great number of dysregulated miRNAs have been discovered in gliomas, which were also found to be involved in multiple crucial biological processes such as proliferation, apoptosis, invasion, differentiation and angiogenesis (8). Importantly, some miRNAs are associated with the grade, prognosis and sensitivity to chemotherapy of gliomas (9). In the present study, we aimed to identify miRNAs involved in tumorigenesis and malignant progression and to conduct preliminary prediction of the potential use of miRNAs in grading and survival expectation. An miRNA microarray approach was used to unravel aberrant miRNA expression in gliomas compared with matched normal tissues. 
Table I. General information regarding the tumor samples for qRT-PCR.

\begin{tabular}{|c|c|c|c|c|}
\hline $\begin{array}{l}\text { Patient } \\
\text { no. }\end{array}$ & Gender & $\begin{array}{c}\text { Age } \\
\text { (years) }\end{array}$ & Grade & Pathology \\
\hline 1 & $\mathrm{~F}$ & 24 & WHO I & DNT \\
\hline 2 & $\mathrm{~F}$ & 17 & WHO I & PA \\
\hline 3 & $\mathrm{~F}$ & 36 & WHO II & $\mathrm{AO}$ \\
\hline 4 & M & 5 & WHO II & A \\
\hline 5 & $\mathrm{M}$ & 38 & WHO II & A \\
\hline 6 & M & 43 & WHO II & A \\
\hline 7 & M & 71 & WHO II & A \\
\hline 8 & $\mathrm{M}$ & 43 & WHO II & $\operatorname{PrA}$ \\
\hline 9 & $\mathrm{~F}$ & 38 & WHO III & $\mathrm{AO}$ \\
\hline 10 & $\mathrm{M}$ & 34 & WHO III & $\mathrm{AO}$ \\
\hline 11 & M & 49 & WHO III & A \\
\hline 12 & $\mathrm{M}$ & 66 & WHO III & $\mathrm{AO}$ \\
\hline 13 & $\mathrm{M}$ & 34 & WHO III & $\mathrm{AO}$ \\
\hline 14 & M & 30 & WHO III & A \\
\hline 15 & $\mathrm{~F}$ & 41 & WHO III & A \\
\hline 16 & $\mathrm{~F}$ & 37 & WHO III & A \\
\hline 17 & M & 49 & WHO III & A \\
\hline 18 & $\mathrm{~F}$ & 42 & WHO III & A \\
\hline 19 & $\mathrm{M}$ & 57 & WHO IV & GBM \\
\hline 20 & $\mathrm{M}$ & 67 & WHO IV & GBM \\
\hline 21 & $\mathrm{M}$ & 63 & WHO IV & GBM \\
\hline 22 & M & 58 & WHO IV & GBM \\
\hline 23 & M & 62 & WHO IV & GBM \\
\hline 24 & $\mathrm{~F}$ & 62 & WHO IV & GBM \\
\hline 25 & M & 66 & WHO IV & GBM \\
\hline 26 & M & 67 & WHO IV & GBM \\
\hline
\end{tabular}

M, male; F, female; A, astrocytoma; PA, pilocytic astrocytoma; PrA, protoplasmic astrocytoma; AO, anaplastic oligodendrogliomas; GBM, glioblastoma multiforme; DNT, dysembryoplastic neuroepthelial tumor; WHO, World Health Organization.

\section{Materials and methods}

Clinical samples. The present study included 26 cases of gliomas including 2, 6, 10 and 8 samples in grade of WHO I-IV, respectively, according to the 2007 WHO criteria (Table I). Among them, 9 cases were used for microarray, which had matched adjacent non-cancerous tissues, and each grade of WHO II, III and IV had 3 patients. The non-neoplastic brain tissues located $>2 \mathrm{~cm}$ from the tumor tissues were obtained (10). All of the patients had no history of radiotherapy or chemotherapy. The samples were collected at the time of surgery at the Department of Neurosurgery, Xinqiao Hospital, Third Military Medical University (TMMU), Chongqing, China, and then immediately stored at $-80^{\circ} \mathrm{C}$ until use. Written informed consents were obtained from all of the patients or their relatives. The present study was approved by the Ethics Committee of Xinqiao Hospital, TMMU. Clinical information
Table II. General information regarding the tumor samples for miRNA microarray analysis.

\begin{tabular}{lcclc}
\hline Patient ID. & Gender & $\begin{array}{c}\text { Age } \\
\text { (years) }\end{array}$ & Grade & Pathology \\
\hline 04050361 & M & 71 & WHO II & A \\
04784847 & M & 38 & WHO II & A \\
04719577 & M & 43 & WHO II & PrA \\
04103496 & M & 49 & WHO III & A \\
03938319 & M & 34 & WHO III & AO \\
03954349 & F & 38 & WHO III & AO \\
04051023 & M & 67 & WHO IV & GBM \\
04653022 & M & 62 & WHO IV & GBM \\
04714692 & F & 62 & WHO IV & GBM
\end{tabular}

M, male; F, female; A, astrocytoma; PrA, protoplasmic astrocytoma; $\mathrm{AO}$, anaplastic oligodendrogliomas; GBM, glioblastoma multiforme; WHO, World Health Organization.

of the patients with tumors and adjacent tissues for microarray assay is summarized in Table II.

miRNA microarray assay. Total RNA was isolated from tissues using the TRIzol method (Life Technologies, USA), according to the manufacturer's protocol. NanoDrop ${ }^{\circledR}$ ND-1000 spectrophotometer (Thermo Fisher Scientific Inc., Wilmington, DE, USA) was used to determine the purity and quantity of total RNA. In order to assess RNA integrity, Agilent RNA 6000 Nano assay (Agilent Technologies, Inc., Santa Clara, CA, USA) and agarose gel electrophoresis, which also checks for genomic DNA contamination, were used. DNase digestion was performed to eliminate DNA found on the gel. Small RNA molecules $(<200 \mathrm{nt})$ were separated from large RNA molecules by NanoSep ${ }^{\circledR} 100 \mathrm{~K}$, based on the filtrate and labeled with cy3/cy5 by the platinum complex of the ULS molecule, which binds to the N7 position of RNA guanine tightly. The labeled RNA was hybridized to the Human miRNA OneArray microarray (Phalanx Biotech Group, Taiwan) containing 1,926 miRNA probes, and the array was later scanned by Axon 4000B (Axon Instruments, Union City, CA, USA) from Molecular Devices.

Microarray data analysis. The data analysis of gene expression profiling, including data filtering, normalization and statistical calculations, was processed by $\mathrm{R}$ (R-Foundation for Statistical Computing, Vienna, Austria; version 2.12.1). Arrays (including technical replicates) of any compared sample set were normalized together after filtering probes. The expression level of each miRNA was calculated by pair-wise combination and error weighted average. The significant differential expression of miRNAs between 9 tumors and 9 matched adjacent noncancerous tissues was selected according to fold-change and a P-value with the following criteria: fold-change $\geq 1.5$ or $\leq 0.67$ and P-value $\leq 0.05$ (11).

Quantitative RT-PCR. Quantitative reverse transcription-PCR (qRT-PCR) was performed for verification of miRNA 
microarray results using 3 selected miRNAs (miR-20a, miR-21 and miR-4489). A total of $1 \mu \mathrm{g}$ of RNA was reversetranscribed into cDNA using the All-in-One ${ }^{\mathrm{TM}}$ miRNA First-strand cDNA synthesis kit (AMRT-0600; GeneCopoeia, Rockville, MD, USA) according to the manufacturer's instructions. Next, the resulting cDNA (1:5 dilution) was amplified and assayed by qPCR, with the 7500 Real-Time PCR system (Applied Biosystems). Specific primers of the miRNAs (HmiRQP0312, HmiRQP0316, HmiRQP2129 and HmiRQP9001) were provided by GeneCopoeia. All PCR reactions were analyzed in triplicate. Relative quantification for each sample was calculated using the $2^{-\Delta \Delta \mathrm{Ct}}$ method, with the universally expressed small nuclear RNA U6 used as the endogenous control. A pooled human brain cerebral cortex total RNA (Clontech, USA) was used as the normal control.

miRNA target prediction and functional analysis. Some online software programs were used for target gene prediction of miRNAs, including miRanda (http://www.microrna. org/microrna/home.do), PICTAR (http://www.ncrna. org/KnowledgeBase/link-database/mirna_target_database) and TargetScan (http://www. targetscan.org). To reduce the number of false positives, only miRNA target genes identified by all of these programs were retained. Predicted target genes were imported into DAVID v6.7, a web-based tool for annotation, visualization and integrated discovery, for functional analysis. We performed biological process of Gene Ontology (GO) and Pathway analysis on target genes of the miRNAs with differential expression based on the GO database and KEGG database. The statistical significance of $\mathrm{GO}$ and pathways was determined on the basis of a P-value $<0.01$.

Statistical analysis. The significance of differences between the compared groups, gliomas and the matched adjacent noncancerous tissues performed by qRT-PCR, was determined by a paired samples t-test using SPSS 16.0 (IBM Corporation, USA) with a P-value of $<0.05$ considered to indicate a statistically significant result.

\section{Results}

miRNA microarray analysis. In the microarray data analysis, we investigated 1,926 miRNAs. Thirteen human miRNAs were significantly differentially expressed between 9 gliomas and the adjacent tissues (fold-change $\geq 1.5$ or $\leq 0.67 ; \mathrm{P} \leq 0.05$ ). Among these, 12 miRNAs were upregulated and 1 was downregulated. Seven miRNAs (hsa-miR-92a-3p, hsa-miR-106b-5p, hsa-miR93-5p, hsa-miR-20a-5p, hsa-miR-17-5p, hsa-miR-25-3p and hsa-miR-19b-3p) are members of the miR-17-92 cluster and its paralogs, miR-106b-25 cluster (Fig. 1). miRNAs encoded by the miR-17-92 cluster show widespread enforced expression and are known as oncogenes in different types of cancers in previous studies $(12,13)$. The cluster analyses revealed complete separation of the patient and control groups based on the expression profiles of the differentially expressed miRNAs (Fig. 2). In addition, by analysis of aberrant expression of miRNAs in WHO II, III and IV compared with the matched adjacent non-cancerous tissues, each grade containing 3 glioma samples, we found 7 upregulated and 1 downregu-
Table III. Aberrantly expressed miRNAs in different grades of gliomas.

\begin{tabular}{lcc}
\hline miRNAs & Ratio (T/N) & P-value \\
\hline WHO II & & \\
hsa-miR-92a-3p & 2.962 & 0.044 \\
hsa-miR-335-5p & 3.634 & 0.042 \\
hsa-miR-18a-5p & 1.812 & 0.032 \\
hsa-miR-19b-3p & 2.335 & 0.001 \\
hsa-miR-3653 & 1.684 & 0.050 \\
hsa-miR-1247-3p & 1.505 & 0.041 \\
hsa-miR-4632-5p & 1.766 & 0.034 \\
hsa-miR-204-3p & 0.437 & 0.014 \\
WHO III & & \\
hsa-miR-125b-5p & 1.772 & 0.041 \\
hsa-miR-106b-5p & 1.958 & 0.018 \\
hsa-miR-99a-3p & 1.582 & 0.017 \\
hsa-miR-93-5p & 1.946 & 0.013 \\
hsa-miR-17-5p & 1.962 & 0.015 \\
hsa-miR-320a & 1.760 & 0.024 \\
hsa-miR-29b-3p & 0.473 & 0.043 \\
hsa-miR-29c-3p & 0.403 & 0.044 \\
hsa-miR-4433-5p & 0.604 & 0.031 \\
WHO IV & & \\
hsa-miR-32-3p & 1.639 & 0.021 \\
hsa-miR-1275 & 1.931 & 0.035 \\
hsa-miR-3178 & 1.861 & 0.004 \\
hsa-miR-3940-5p & 1.631 & 0.036 \\
hsa-miR-4725-3p & 2.073 & 0.018 \\
hsa-miR-642b-3p & 1.580 & 0.022 \\
hsa-miR-138-5p & 0.396 & 0.019 \\
hsa-miR-29b-2-5p & 0.658 & 0.010 \\
hsa-miR-139-5p & 0.353 & 0.037 \\
hsa-miR-3144-5p & 0.587 & 0.016 \\
hsa-miR-3161 & 0.622 & 0.024 \\
hsa-miR-4526 & 0.631 & 0.040 \\
hsa-miR-4525 & 0.496 & 0.022 \\
hsa-miR-4694-3p & 0.665 \\
hsa-miR-6501-3p & 0.605 & 0.032 \\
\hline T, & & 0.012 \\
\hline
\end{tabular}

$\mathrm{T}$, tumor; $\mathrm{N}$, non-cancerous tissue surrounding the tumor; $\mathrm{WHO}$, World Health Organization.

lated miRNA in WHO II, 6 upregulated and 3 downregulated miRNAs in WHO III and 6 upregulated and 9 downregulated miRNAs in WHO IV (Table III).

miRNA expression validation. To improve sample representativeness, 3 candidate miRNAs, miR-20a-5p (randomly selected), miR-21-5p (the common upregulated) and miR-4489 (the only one downregulated), were selected to validate the microarray data by quantitative RT-PCR assay using the same RNA samples that were used for the microarrays. The adjacent non-cancerous tissues acted as the control in the same 
\begin{tabular}{l|l}
$\operatorname{miR}-106 b-25$ & hsa-miR-25-3p \\
(7q22.1) & $\begin{array}{l}\text { hsa-miR-93-5p } \\
\text { hsa-miR-106b-5p }\end{array}$
\end{tabular}

miR-17-92

(13q31.3)

hsa-miR-17-5p
hsa-miR-20a-5p
hsa-miR-19b-3p
hsa-miR-92a-3p

\section{AGGCGGAGACUUGGGCAAUUG \\ CAAAGUGCUGUUCGUGCAGGUAG \\ UAAAGUGCUGACAGUGCAGAU}

\section{CAAAGUGCUUACAGUGCAGGUAG \\ CAAAGUGCUCAUAGUGCAGGUAG \\ UGUGCAAAUCCAUGCAAAACUGA \\ UAUUGCACUUGUCCCGGCCUGU}

Figure 1. Characteristics of members in the miR-17-92 and miR-106b-25 clusters. A total of 7 positive miRNAs are involved in the miR-17-92 and miR-106b-25 clusters. Four of them have the same seed sequences, which are the regions considered most important for candidate targets (nucleotides 2-7; shown in red).

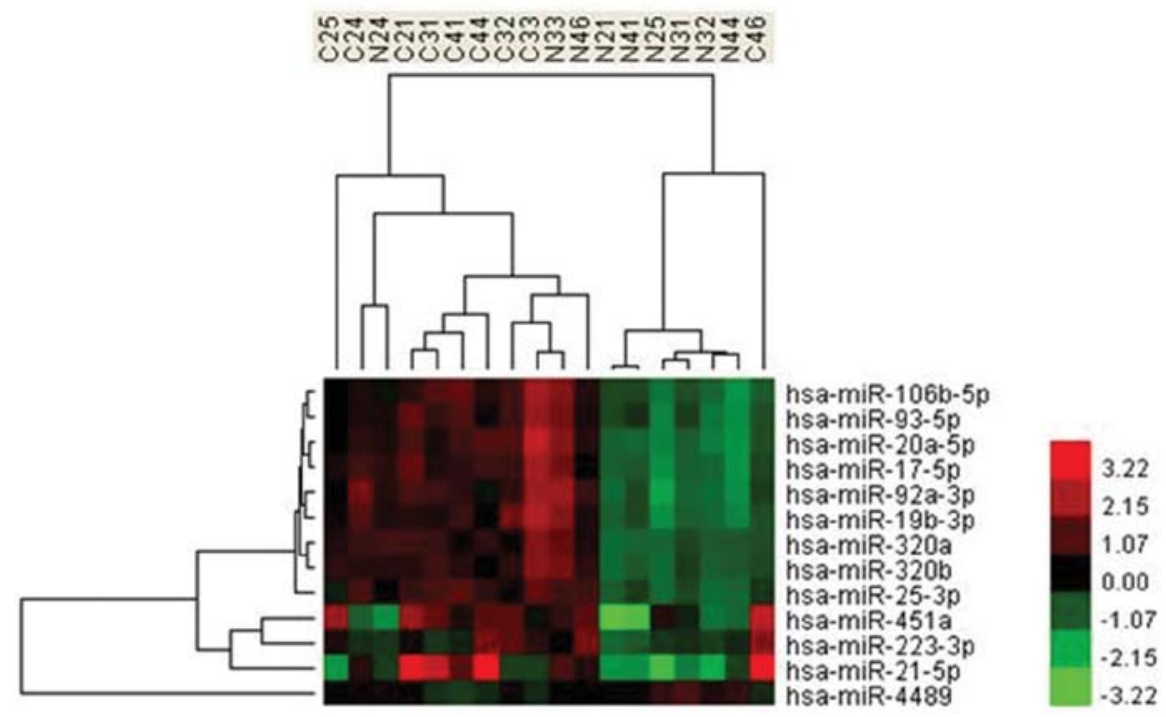

Figure 2. A hierarchical clustering of dysregulated miRNAs. Results of the cluster analysis indicate the altered microRNA expression pattern of gliomas compared with the corresponding normal tissues.

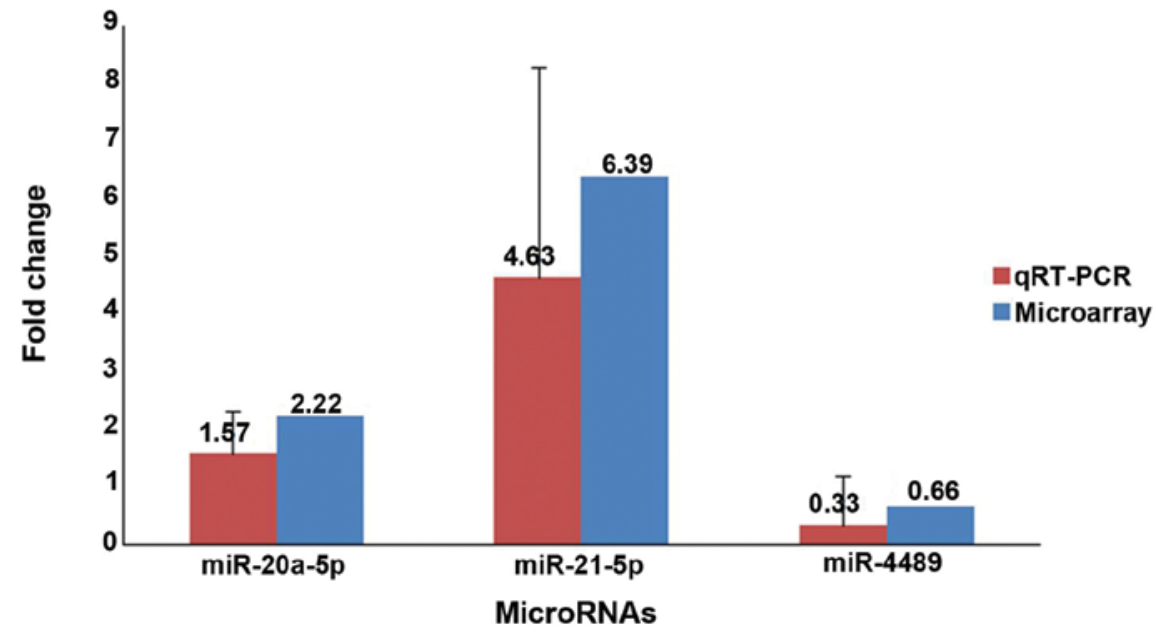

Figure 3. Comparison of miRNA expression levels between the miRNA microarray and qRT-PCR analysis. Microarray and qRT-PCR results for miR-20a-5p, miR-21-5p and miR-4489 were obtained from 9 pairs of glioma samples and the matched adjacent normal tissues. The values of the adjacent normal tissues were set at 1 for both experiments. As shown, the fold-changes in microRNA expression levels detected by qRT-PCR were closely correlated with those by microarray.

groups for both experiments. Compared with the adjacent non-cancerous tissues, the expression levels of miR-20a-5p $(\mathrm{P}=0.049, \mathrm{n}=9)$ and miR-21-5p $(\mathrm{P}=0.017, \mathrm{n}=9)$ were present in higher abundance, whereas miR-4489 $(\mathrm{P}=0.045, \mathrm{n}=9)$ was present in lower abundance in the gliomas. The results of quantitative RT-PCR were in accordance with the miRNA 


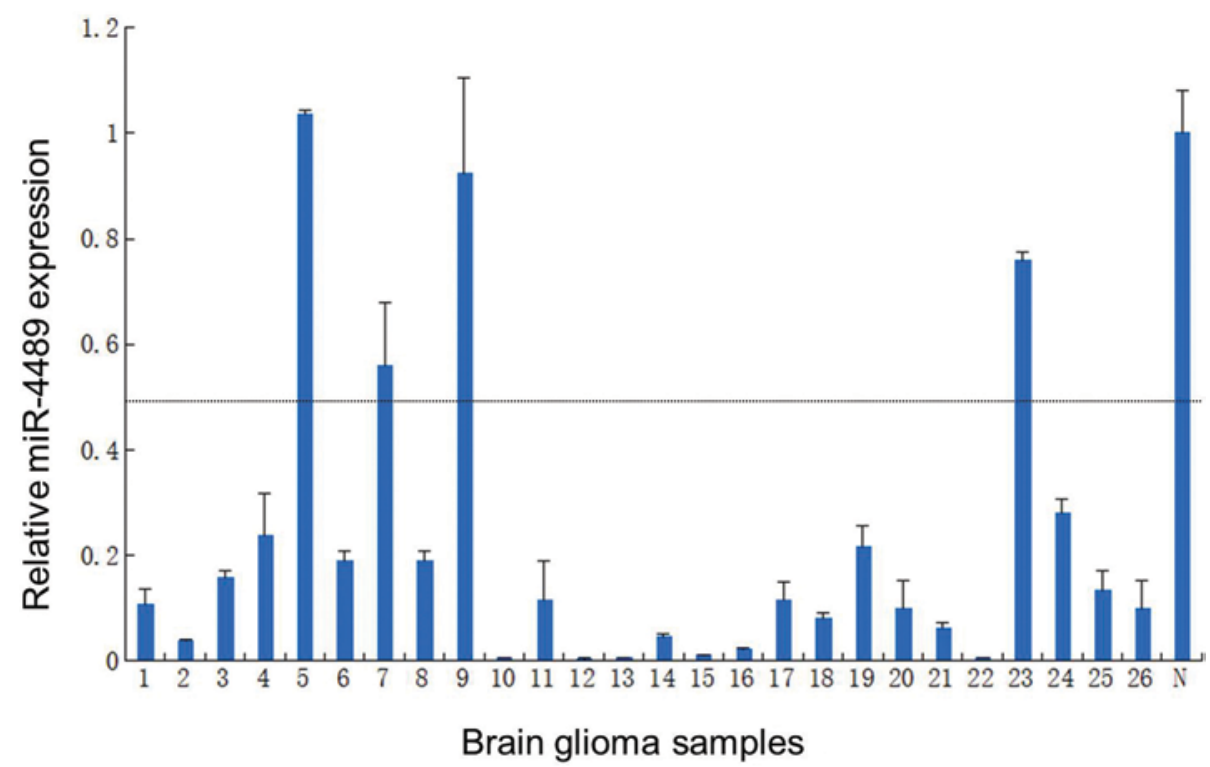

Figure 4. Downregulation of miR-4489 in brain glioma samples. Relative miR-4489 expression in brain glioma samples as determined by qRT-PCR. Values represent the fold of miR-4489 mRNA relative to normal cerebral cortex total RNA (Clontech, USA) (n=3, \pm SD). Glioma samples (22 of 26) (84.62\%) displayed miR-4489 expression $<50 \%$ of the human normal cerebral cortex $(\mathrm{N})$.

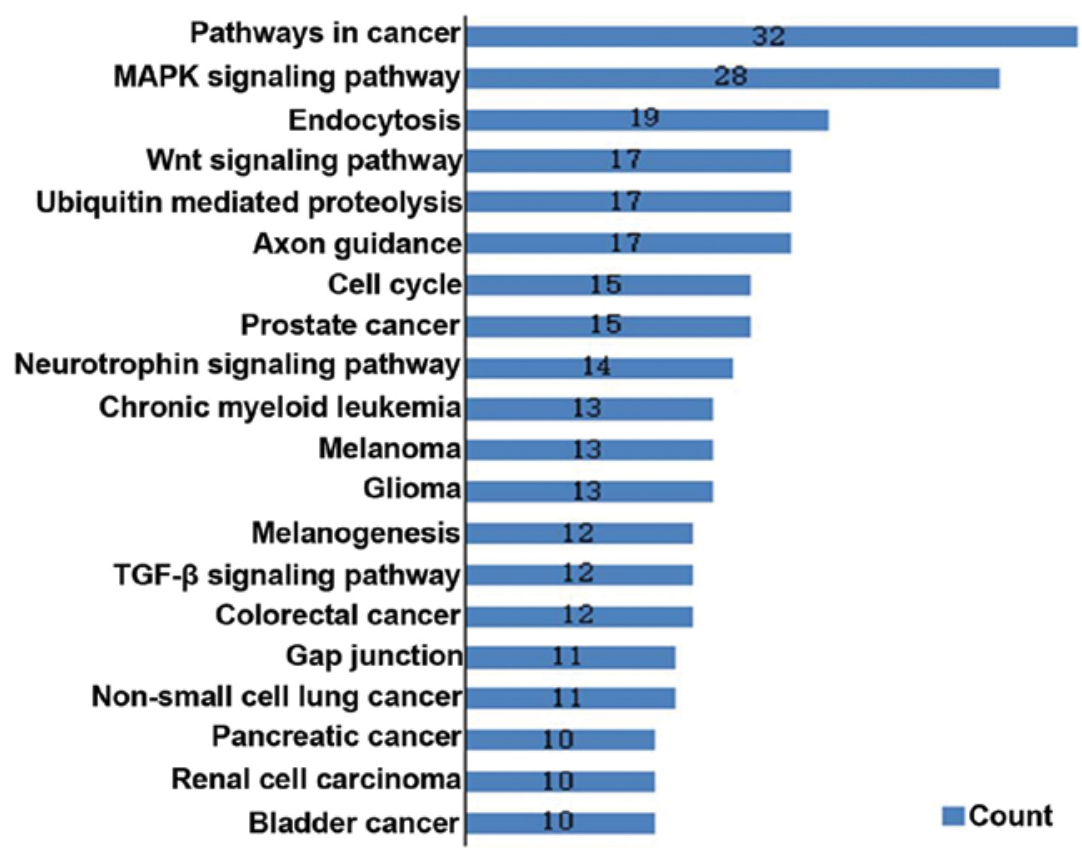

Figure 5. KEGG pathway analysis for target genes of the dysregulated miRNAs. Potential target genes of 13 miRNAs were enriched in tumor-related pathways including MAPK, the cell cycle and the Wnt signaling pathway. Each bar in blue indicates the number of target genes involved in the relevant pathway.

microarray results (Fig. 3). Furthermore, we investigated the expression of miR-4489 by qRT-PCR in 26 glioma samples. The results presented in Fig. 4 revealed that $84.62 \%$ (22/26) of gliomas had a reduced miR-4489 expression compared to the normal human cerebral cortex total RNA (Clontech).

miRNA target gene prediction, GO and Pathway analysis. The potential target genes of the 13 dysregulated miRNAs were predicted by different algorithms, including TargetScan, PICTAR and miRanda. Only miRNA target genes identified by all of these algorithms were considered. Some of the
miRNAs, for example, miR-4489 were only included in the database of TargetScan; hence, target genes of miR-4489 in TargetScan were selected as a part of the target gene set for further analysis. A total of 926 target genes were predicted. Target genes (720) and 162 significant categories were found in Gene Ontology Biological Process when GO analysis was performed in the DAVID database (DAVID Bioinformatics Resources 6.7). A large number of target genes were involved in the enriched GO categories notably genes involved in the regulation of cell proliferation, the cell cycle, apoptosis and neuronal differentiation. Pathway analysis based on the KEGG 
pathway database identified 239 target genes and 20 enriched pathways $(\mathrm{P}<0.01)($ Fig. 5).

\section{Discussion}

In the past 9 years, extensive research has indicated that miRNAs contribute to the development and progression of gliomas (8). However, the expression patterns of grade-specific miRNAs that may act as reliable biomarkers for early diagnosis, progression detection and prediction of survival are largely unknown. In the present study, using an miRNA microarray approach, we uncovered the aberrant expression of several miRNAs in gliomas of different grades compared with the adjacent tissues. In previous studies using microarrays, normal tissues and brain tumors were selected from different patients (14). However, in the present study, the gliomas and non-neoplasms were selected from the same patients concurrently, and this effectively reduced the impact of individual differences. We selected 3 pairs of samples in each grade of WHO II, III and IV for miRNA microarray analysis. Totals of 8,9 and 15 dysregulated miRNAs were detected in WHO II, III and IV gliomas, respectively, and the miRNAs in each grade of gliomas were entirely different from those in the other grades. Aberrantly expressed miRNAs only appeared in a certain grade but were absent in the lower grades, indicating that these miRNAs result in the malignant progression of gliomas. These may also be used as valuable biomarkers for glioma grading. As a single mRNA can be regulated by multiple miRNAs, an association would be found in the level of protein or mRNA despite the apparent unrelatedness of miRNAs in each grade. In addition, a majority of our miRNAs, such as miR-4433-5p and miR-4725-3p, have not been involved in other studies. These miRNAs may serve as important resources for future functional study in gliomas.

Thirteen human miRNAs were found to be significantly differentially expressed between gliomas and the adjacent tissues in an integrated analysis. A total of 926 predicted target genes of the 13 miRNAs were obtained by bioinformatics. GO and Pathway analysis showed that a large number of target genes were significantly enriched in biological processes and signaling pathways associated with the nervous system and tumors. Several cancer-related pathways, including the MAPK, p53, Wnt signaling pathways and cell cycle-related pathway, were included in the enriched pathways of the target genes. Aberrant activation of Wnt signaling, which increases cell proliferation and enhances invasiveness, is involved in various types of cancers, including gliomas (15). MAPK signaling has been considered to contribute to the pathogenesis of gliomas by supporting the migration and invasion of malignant glioma cells (16). TP53 signaling is important in apoptosis and cell cycle arrest in cellular responses to DNA damage and aberrant pathway activation (17,18). Approximately 87\% of glioblastomas harbor at least one component of TP53 signaling aberrations (19). This indicates that aberrant expression of miRNAs in our results contributes to the pathogenesis of gliomas.

Several of the differentially expressed miRNAs, such as miR-21, in our results had previously been demonstrated to be associated with gliomas and to participate in disease development and progression. Chan et al (7) initially found high expression of miR-21 in gliomas by miRNA microarray. In the present study, a 4.63- and 6.39-fold miR-21 expression level in gliomas was detected by qRT-PCR analysis $(\mathrm{P}=0.017, \mathrm{n}=9)$ and microarray $(\mathrm{P}=0.028, \mathrm{n}=9)$, respectively, compared with the adjacent non-cancerous tissues (Fig. 3). Moreover, our results are in accordance with Chan et al. The upregulation of miR-21 was found to increase cell growth by inhibiting PDCD4 (20) and to enhance invasiveness by inhibiting SPYR2 (21). Moreover, Han et al (22) identified that the miR-21 expression level increased with an increasing histological grade of gliomas. A novel dysregulated miRNA, miR-4489, was found to be related to human gliomas in the present study. However, the significance of dysregulated expression of miR-4489 was not found in WHO II, III and IV in the microarray (Table III). To further detect the expression of miRNA-4489, 26 samples were used for qRT-PCR method compared with human normal cerebral cortex (Clontech). In the present study, 84.62\% (22/26) of glioma samples showed downregulation of miR-4489 by qRT-PCR. A total of 89 potential target genes for miR-4489 were obtained from the TargetScan database. According to the result of the GO analysis, a number of target genes were enriched in the process of cell proliferation. Among these, several genes such as CDK6 were verified to be correlated with gliomas (23). Analyses of clinical samples from GBM patients identified a higher CDK6 antigen expression in tumors than in adjacent normal brain tissues. Knockdown of CDK6 by siRNAs induced cell cycle arrest at the G1/S transition and inhibited cell proliferation. In addition, TGF- $\beta 2$, a predicted target of miR-4489, is a key factor in the pathogenesis of gliomas, and is involved in tumor growth, invasion and metastasis (24). Bioinformatics software provided a primary analysis of miR-4489 functions and target genes, and this contributes to new clues for research on gliomas.

The miR-17-92 cluster (miR-17, miR-18a, miR-19a, miR-20a, miR-19b-1 and miR-92-1) is a typical case known as oncogenes in diverse tumor subtypes. However, as a paralog of the miR-17-92 cluster, the function of the miR-106b-25 cluster, including 3 miRNAs (miR-106b, miR-93 and miR-25), remains largely unclear in carcinogenesis. In the present study, all members of the miR-106b-25 cluster and 4 miRNAs in the miR-17-92 cluster were included in the aberrantly expressed miRNAs. Ventura et al (25) showed that the functions of the miR-106b-25 cluster are seemingly dispensable in normal mouse embryonic development and is the only study in the context of miR-17-92 cluster loss. In our data, the aberrant expression of members in the miR-17-92 cluster (miR-92a-3p, miR-18a-5p and miR-19b-3p) was found in WHO II gliomas, while dysregulated expression of miRNAs in the miR-106b-25 cluster (miR-106b-5p and miR-93-5p) was detected in WHO III gliomas. Two members of the miR-106b-25 cluster have an identical seed sequence (nucleotides 2-7), which is also the seed sequence of two members of the miR-17-92 cluster (Fig. 1). The highly conserved feature of this region indicates the functional importance of these miRNAs. However, according to the results of prevailing target prediction models, it is also reasonable to suppose that these miRNAs should be functionally redundant. As observed during normal development of mice, enhanced expression of the miR-106b-25 cluster plays an assistant role in glioma progression and results in greater proliferation ability and anti-apoptotic effects in tumors 
with upregulated expression levels of the miR-17-92 cluster. There is reason to believe that the miR-106b-25 cluster plays an indispensable role in the malignant process of gliomas by promoting cell cycle progression, enhancing cell proliferation, inhibiting cell apoptosis and inducing blood vessel formation (26-29). Despite the great progress made in understanding the cluster's roles, the expression pattern and the precise role of each miRNA in gliomas need to be deeply elucidated. Further study of the function of the miR-106b-25 cluster may provide new insight into the role of miRNAs in the development and progression of human gliomas.

To summarize, we described the differential expression of miRNAs and explored the grade-related miRNA expression profiles in brain gliomas. These miRNAs could disrupt crucial pathophysiological processes. These results have significant importance in uncovering a relationship between specific miRNAs and glioma tumorigenesis, malignant progression and different glioma grades.

\section{Acknowledgements}

We thank Dr Kaifeng Shen and Mrs. Zhenle Zang for their technical assistance. The present study was supported by the National Natural Science Foundation of China (NSFC: 81272783), The Natural Science Foundation Project of CQ (CSTC: 2010BB5028) and The National Key Technology Research and Development Program of the Ministry of Science and Technology of China (2014BAI04B02).

\section{References}

1. Kanzawa T, Germano IM, Komata T, Ito H, Kondo $\mathrm{Y}$ and Kondo S: Role of autophagy in temozolomide-induced cytotoxicity for malignant glioma cells. Cell Death Differ 11: 448-457, 2004.

2. Stupp R, Mason WP, van den Bent MJ, Weller M, Fisher B, Taphoorn MJ, Belanger K, Brandes AA, Marosi C, Bogdahn U, et al; European Organisation for Research and Treatment of Cancer Brain Tumor and Radiotherapy Groups; National Cancer Institute of Canada Clinical Trials Group: Radiotherapy plus concomitant and adjuvant temozolomide for glioblastoma. $\mathrm{N}$ Engl J Med 352: 987-996, 2005.

3. Louis DN, Ohgaki H, Wiestler OD, Cavenee WK, Burger PC, Jouvet A, Scheithauer BW and Kleihues P: The 2007 WHO classification of tumours of the central nervous system. Acta Neuropathol 114: 97-109, 2007.

4. Ohgaki $\mathrm{H}$ and Kleihues P: The definition of primary and secondary glioblastoma. Clin Cancer Res 19: 764-772, 2013.

5. Ambros V: microRNAs: Tiny regulators with great potential. Cell 107: 823-826, 2001.

6. Lu J, Getz G, Miska EA, Alvarez-Saavedra E, Lamb J, Peck D, Sweet-Cordero A, Ebert BL, Mak RH, Ferrando AA, et al: MicroRNA expression profiles classify human cancers Nature 435: 834-838, 2005

7. Chan JA, Krichevsky AM and Kosik KS: MicroRNA-21 is an antiapoptotic factor in human glioblastoma cells. Cancer Res 65: 6029-6033, 2005

8. Davis-Dusenbery BN and Hata A: MicroRNA in cancer: The involvement of aberrant microRNA biogenesis regulatory pathways. Genes Cancer 1: 1100-1114, 2010.

9. He J, Deng Y, Yang G and Xie W: MicroRNA-203 down-regulation is associated with unfavorable prognosis in human glioma. J Surg Oncol 108: 121-125, 2013.

10. Yan LX, Huang XF, Shao Q, Huang MY, Deng L, Wu QL, Zeng YX and Shao JY: MicroRNA miR-21 overexpression in human breast cancer is associated with advanced clinical stage, lymph node metastasis and patient poor prognosis. RNA 14: 2348-2360, 2008.
11. He HC, Han ZD, Dai QS, Ling XH, Fu X, Lin ZY, Deng YH, Qin GQ, Cai C, Chen JH, et al: Global analysis of the differentially expressed miRNAs of prostate cancer in Chinese patients. BMC Genomics 14: 757, 2013.

12. Cho WC: OncomiRs: The discovery and progress of microRNAs in cancers. Mol Cancer 6: 60, 2007.

13. Diosdado B, van de Wiel MA, Terhaar Sive Droste JS, Mongera S, Postma C, Meijerink WJ, Carvalho B and Meijer GA: MiR-17-92 cluster is associated with $13 \mathrm{q}$ gain and c-myc expression during colorectal adenoma to adenocarcinoma progression. $\mathrm{Br} \mathrm{J}$ Cancer 101: 707-714, 2009.

14. Li Y, Xu J, Chen H, Bai J, Li S, Zhao Z, Shao T, Jiang T, Ren H, Kang C, et al: Comprehensive analysis of the functional microRNA-mRNA regulatory network identifies miRNA signatures associated with glioma malignant progression. Nucleic Acids Res 41: e203, 2013.

15. Wang K, Wang X, Zou J, Zhang A, Wan Y, Pu P, Song Z, Qian C, Chen Y, Yang S, et al: miR-92b controls glioma proliferation and invasion through regulating $\mathrm{Wnt} /$ beta-catenin signaling via Nemo-like kinase. Neuro-oncol 15: 578-588, 2013

16. Guo G, Yao W, Zhang Q and Bo Y: Oleanolic acid suppresses migration and invasion of malignant glioma cells by inactivating MAPK/ERK signaling pathway. PLoS One 8: e72079, 2013.

17. Kruse JP and Gu W: Modes of p53 regulation. Cell 137: 609-622, 2009.

18. Farnebo M, Bykov VJ and Wiman KG: The p53 tumor suppressor: A master regulator of diverse cellular processes and therapeutic target in cancer. Biochem Biophys Res Commun 396: 85-89, 2010.

19. Cancer Genome Atlas Research Network: Comprehensive genomic characterization defines human glioblastoma genes and core pathways. Nature 455: 1061-1068, 2008.

20. Gaur AB, Holbeck SL, Colburn NH and Israel MA: Downregulation of Pdcd 4 by mir-21 facilitates glioblastoma proliferation in vivo. Neuro-oncol 13: 580-590, 2011.

21. Kwak HJ, Kim YJ, Chun KR, Woo YM, Park SJ, Jeong JA, Jo SH, Kim TH, Min HS, Chae JS, et al: Downregulation of Spry2 by miR-21 triggers malignancy in human gliomas. Oncogene 30 : 2433-2442, 2011

22. Han L, Yue X, Zhou X, Lan FM, You G, Zhang W, Zhang KL, Zhang CZ, Cheng JQ, Yu SZ, et al: MicroRNA-21 expression is regulated by $\beta$-catenin/STAT3 pathway and promotes glioma cell invasion by direct targeting RECK. CNS Neurosci Ther 18: 573-583, 2012.

23. Chen SM, Chen HC, Chen SJ, Huang CY, Chen PY, Wu TW, Feng LY, Tsai HC, Lui TN, Hsueh C, et al: MicroRNA-495 inhibits proliferation of glioblastoma multiforme cells by downregulating cyclin-dependent kinase 6. World J Surg Oncol 11: 87, 2013.

24. Wick W, Naumann U and Weller M: Transforming growth factorbeta: A molecular target for the future therapy of glioblastoma. Curr Pharm Des 12: 341-349, 2006.

25. Ventura A, Young AG, Winslow MM, Lintault L, Meissner A Erkeland SJ, Newman J, Bronson RT, Crowley D, Stone JR, et al: Targeted deletion reveals essential and overlapping functions of the miR-17 through 92 family of miRNA clusters. Cell 132: $875-886,2008$

26. Kan T, Sato F, Ito T, Matsumura N, David S, Cheng Y, Agarwal R, Paun BC, Jin Z, Olaru AV, et al: The miR-106b-25 polycistron, activated by genomic amplification, functions as an oncogene by suppressing p21 and Bim. Gastroenterology 136: 1689-1700, 2009.

27. Ivanovska I, Ball AS, Diaz RL, Magnus JF, Kibukawa M, Schelter JM, Kobayashi SV, Lim L, Burchard J, Jackson AL, et al: MicroRNAs in the miR-106b family regulate p21/CDKN1A and promote cell cycle progression. Mol Cell Biol 28: 2167-2174, 2008.

28. Liu F, Gong J, Huang W, Wang Z, Wang M, Yang J, Wu C, Wu Z and Han B: MicroRNA-106b-5p boosts glioma tumorigensis by targeting multiple tumor suppressor genes. Oncogene 33 : 4813-4822, 2014.

29. Fang L, Deng Z, Shatseva T, Yang J, Peng C, Du WW, Yee AJ, Ang LC, He C, Shan SW, et al: MicroRNA miR-93 promotes tumor growth and angiogenesis by targeting integrin- $\beta 8$. Oncogene 30: 806-821, 2011. 